\title{
Development and Validation of a Rapid and Reliable RP-HPLC-PDA Method for the Quantification of Six Diterpenes in Copaifera duckei, Copaifera reticulata and Copaifera multijuga Oleoresins
}

\author{
Luiza J. Carneiro, ${ }^{a}$ Thamires C. Bianchi, ${ }^{a}$ Jonas J. M. da Silva, ${ }^{b}$ Larissa C. Oliveira, ${ }^{a}$ \\ Carly H. G. Borges, ${ }^{a}$ Danieli C. Lemes, ${ }^{a}$ Jairo K. Bastos, ${ }^{b}$ Rodrigo C. S. Veneziani ${ }^{*, a}$ and \\ Sérgio R. Ambrósio*,a
}

${ }^{a}$ Núcleo de Pesquisa em Ciências Exatas e Tecnológicas, Universidade de Franca, Av. Dr. Armando de Salles Oliveira, 201, 14404-600 Franca-SP, Brazil

${ }^{b}$ Faculdade de Ciências Farmacêuticas de Ribeirão Preto, Universidade de São Paulo, Av. do Café, s/n, 14040-903 Ribeirão Preto-SP, Brazil

\begin{abstract}
Oleoresins from Copaifera species are extensively used in folk medicine in Brazil, which are employed mainly in the production of cosmetic formulations in Brazil, North America and Europe. Considering the lack of validated analytical methods for the analyses of diterpenes in Copaifera oleoresins, it was developed a validated and reliable reversed-phase high-performance liquid chromatography with photodiode array detection (RP-HPLC-PDA) method for the analysis of six diterpenes, including: (-)-polyalthic acid; (13E)-ent-labda-7,13-dien-15-oic acid; ent-8(17)-labden-15,18-dioic acid; (-)-copalic acid; (-)-3 $\beta$-acetoxycopalic acid and (-)-3ß-hydroxycopalic acid. These compounds were isolated from C. duckei, C. reticulata and C. multijuga oleoresins by chromatographic means. The analytical curves were linear with regression coefficients $\left(\mathrm{r}^{2}\right)$ between 0.9903 and 0.9999 . The limits of detection (LOD) and quantification (LOQ) values were 0.35 to $3.09 \mu \mathrm{g} \mathrm{mL} \mathrm{mL}^{-1}$ and 1.05 to $9.36 \mu \mathrm{g} \mathrm{mL} \mathrm{mL}^{-1}$, respectively. The method also displayed good precision and accuracy. The developed analytical method is reliable and a useful tool for the analysis of Copaifera oleoresin and its products.
\end{abstract}

Keywords: Copaifera spp., oleoresins, diterpenes, RP-HPLC-PDA

\section{Introduction}

The Copaifera genus (Leguminoseae) consists of approximately 72 species, from which 16 of them occur only in Brazil, mainly in Northern region in the states of Amazonas, Pará and Ceará. ${ }^{1,2}$ These trees are popularly known as "copaiba", "copaibeiras" or "pau d'óleo", and the oleoresins obtained from their trunks are extensively used in folk medicine due to their antiinflammatory, analgesic, wound healing, antimicrobial, antileishmanial and antitumoral properties. ${ }^{2,3}$ Such ethnopharmacological relevance has stimulated several researchers to investigate their biological activities, which corroborated their pharmacological potential. ${ }^{4-8}$ In addition to their pharmaceutical properties, these balsams are also largely used as dietary supplement, employed in the production of flavoring agents, and extensively

*e-mail: rodrigo.veneziani@unifran.edu.br; sergio.ambrosio@unifran.edu.br commercialized as crude oil in Brazil. Moreover, these natural products are exported to Europe and North America, to be used mainly in cosmetic formulations. ${ }^{2,9}$

Despite the great relevance of these oleoresins for pharmaceutical and cosmetic industries, most of these commercial balsams have not been authenticated, and their chemical profile has not been well established, thus hindering their industrial and biomedical applications, as well as impairing the quality and economic value of such products. ${ }^{910}$

Chemically, the oleoresins of copaiba are predominantly composed by a mixture of volatile and non-volatile compounds comprising mainly sesquiterpenes and acid diterpenes. Acid diterpenes stands out for a wide range of the biological properties reported for these oleoresins, such as: antimicrobial, antitumoral, antinociceptive and anti-inflammatory, among others. ${ }^{3,4,6,11-15}$ Despite this fact, most of the studies reporting the chemical identification and quantification of the diterpenes of Copaifera oleoresins have been performed by using gas chromatographic hyphenated 
techniques and mass spectra comparison with spectral libraries, ${ }^{2,16-20}$ which are more adequate for analyses of volatile compounds like sesquiterpenes. ${ }^{9}$ In the literature, there are only two studies describing the development and validation of analytical methods for the analysis acid diterpenes ${ }^{9,21}$ in Copaifera species. Souza et al..$^{9}$ only comprises the analysis of one standard ((-)-copalic acid) in commercial samples of oleoresins and the study published by Mangabeira et al. ${ }^{21}$ is based on ultra-performance liquid chromatography-tandem mass spectrometer (UPLC-MS/MS).

Based on the need of new analytical validated methods, and as part of our ongoing efforts to explore the potential of Copaifera oleoresins, we are describing, in this paper, the development and validation of a simple and novel analytical method using reversed-phase high-performance liquid chromatography (RP-HPLC). The developed method is capable of quantifying six diterpenes in the non-volatile fractions of the oleoresins obtained from the most commercially explored species: Copaifera duckei, Copaifera reticulata and Copaifera multijuga.

\section{Experimental}

\section{General experimental procedures and reagents}

The liquid chromatography system consisted of a two-pump Shimadzu chromatograph model LC-20A Prominence equipped with a SIL-20A auto sampler, a CTO-20A column oven, a CBM-20A communication bus module, a DGU-20A3 in-line degasser, and an SPD-M20A photodiode array detector (PDA). The LC solution software (version 1.25) was used to process the data. The analyses were conducted on a Shim-pack CLC-ODS column $(250 \times 4.6 \mathrm{~mm}$ i.d., $5 \mu \mathrm{m}$; Shimadzu $)$. Acetonitrile (chromatographic grade) was supplied by Mallinkrodt Baker Inc. (Phillipsburg, NJ, USA). Water was purified with a Milli-Q-plus filter system (Millipore, Bedford, MA, USA). Classic and vacuum liquid chromatography (CC and VLC, respectively; glass columns of $450 \times 25 \mathrm{~mm}$ and 50-100 $\mathrm{mm}$ i.d.) were used to purify the diterpenes by using silica gel 60 (Merck, 9385) and silica gel 60H (Merck, 7736). Commercial hexanes and ethyl acetate (EtOAc) were purified by distillation in our facilities. The nuclear magnetic resonance (NMR) spectra were run on a Bruker DPX 400 spectrometer (400 MHz for ${ }^{1} \mathrm{H}$ and $100 \mathrm{MHz}$ for $\left.{ }^{13} \mathrm{C}\right)$. The samples were dissolved in $\mathrm{CDCl}_{3}$, and the spectra were calibrated with the solvent signals at $7.26\left({ }^{1} \mathrm{H}\right)$ and $77.0\left({ }^{13} \mathrm{C}\right)$. Manool (Figure 1, IS), a labdane diterpene that is not found in Copaifera oleoresins, was used as internal standard (IS) and was acquired from GlycoSyn ( $\geq 95 \%$ purity, Wellington, New Zealand).
Plant material

The studied Copaifera oleoresins were collected in different cities of the Brazilian northern region, as following: C. duckei Dwyer (OCd, Belém, Pará State, 0106.933'S, $\left.48^{\circ} 19.781^{\prime} \mathrm{W}\right)$, C. reticulata Ducke (OCr, Brazil Novo, Pará State, $03^{\circ} 22.028^{\prime} \mathrm{S}, 5^{\circ} 29.947^{\prime} \mathrm{W}$ ), and C. multijuga (OCm, Manacapuru, Amazonas State, $03^{\circ} 11.858^{\prime}$ S , 60³5.437’ W) by Jonas J. M. da Silva. The identifications were carried out by Silvana Tavares Rodrigues from Embrapa, Belém, Pará, and the voucher specimens are deposited in the Embrapa Herbarium, respectively, under the numbers NID:96/2012, NID:03/2013 and NID:62/2013.

Isolation of main diterpenes from the oleoresins

Initially, $100 \mathrm{~g}$ of OCd were chromatographed using vacuum liquid chromatography (VLC system; silica gel $60 \mathrm{H} ; 750 \mathrm{~g})$ with increasing amounts of EtOAc (10\%) using hexanes as eluent, thus furnishing 10 fractions (OCd1-OCd10; $2.0 \mathrm{~L}$ each). Fraction OCd2 (31.1 g) was also fractioned by VLC (silica gel $60 \mathrm{H} ; 750 \mathrm{~g}$ ) with increasing amounts of EtOAc in hexanes (hexanes/EtOAc 99:1 to 9:1) to give ten additional fractions $(400 \mathrm{~mL}$ each; OCd2.1-OCd2.10), which were analyzed through HPLC (acetonitrile/water $+0.1 \%$ acetic acid; $1 \mathrm{~mL} \mathrm{~min}^{-1} ; 201 \mathrm{~nm}$ ). Fractions $\mathrm{OCd} 2.7-\mathrm{OCd} 2.8$ were combined to give the same pure compound $(\mathbf{1} ;(-)$-polyalthic acid; $19.2 \mathrm{~g})$. Fraction OCd3 (1.3 g) was fractionated using classic chromatography (CC; silica gel 60;80 g) and hexane/ethyl acetate 8:2 as mobile phase to give (13E)-ent-labda-7,13-dien-15-oic acid (2; $430.0 \mathrm{mg}$; yield: $0.43 \%$ ). Fractions OCd4 and OCd5 were combined giving a white solid after concentration under vacuum. This solid was washed with cold dichloromethane, thus furnishing ent-8(17)-labden-15,18-dioic acid (3; $8.3 \mathrm{~g}$; yield: $8.3 \%$ ). All these procedures were previously reported by our research group. ${ }^{22}$

Oleoresin of C. multijuga (OCm, $100.0 \mathrm{~g}$ ) was chromatographed on VLC (silica gel $60 \mathrm{H} ; 750 \mathrm{~g}$ ) using increasing amounts of ethyl acetate $(10 \%)$ in hexanes furnishing 11 fractions (OCm1-OCm11). An aliquot of OCm3 (300.0 mg) was fractionated on CC (silica gel 60; $50 \mathrm{~g}$ ) using hexane/ethyl acetate 8:2 (10 mL each fraction) to furnish $203.0 \mathrm{mg}$ of compound 4 ((-)-copalic acid; yield: $0.203 \%$ ). Fractions OCm4 and OCm6 displayed a pure chromatographic profile, leading to the identification of compounds 5 ((-)-3ß-acetoxycopalic acid; $4.3 \mathrm{~g}$; yield: $4.3 \%)$ and $6((-)-3 \beta$-hydroxycopalic acid; $1.3 \mathrm{~g}$; yield: $1.3 \%)$, respectively.

Finally, a VLC column was performed with $100.0 \mathrm{~g}$ of C. reticulata oleoresin (OCr), as described above, furnishing 
11 fractions (OCr1-OCr11). These fractions were also analyzed by HPLC (acetonitrile/water $+0.1 \%$ acetic acid;

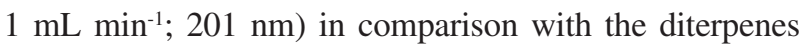
standards isolated from OCd and OCm. (-)-Polyalthic acid (1) was the major constituent in most fractions. Analysis of the chromatogram of $\mathrm{OCr} 3$, and further classic chromatography fractionation using hexanes/ethyl acetate 8:2 as eluent, furnished a mixture of the isomers $\mathbf{2}$ and $\mathbf{4}$.

\section{Obtainment of the oleoresin non-volatile fractions}

The non-volatile oleoresin fractions of $C$. duckei (NVCd), C. multijuga (NVCm) and C. reticulata (NVCr) were obtained from their respective oleoresins through hydrodistillation, as previously described. ${ }^{20}$ Briefly, $50.0 \mathrm{~g}$ of the oleoresin were transferred to a round-bottom flask containing $500 \mathrm{~mL}$ of distilled water, which was attached to a Clevenger-type apparatus. The system was heated and kept under distillation until all the volatile compounds were distilled, in $45 \mathrm{~min}$. The remaining water fraction was then partitioned three times with $500 \mathrm{~mL}$ of ethyl acetate. The resulting organic phases were combined, concentrated under vacuum and named as NVCd (36.3 g), NVCm (3.6 g) and NVCr (29.5 g).

\section{Development and validation of the analytical method}

Chromatographic analyses of NVCd, NVCm and NVCr were performed through RP-HPLC-PDA using a Shim-pack CLC-ODS column $(250 \times 4.6$ mm i.d., $5 \mu \mathrm{m}$; Shimadzu $)$, and an isocratic system consisting of acetonitrile and water with $0.1 \%$ acetic acid (8:2 v/v) as mobile phase, which was established through initial experiments using the gradient scouting run evaluation as previously described. ${ }^{23}$ The temperature of the column was set at $40{ }^{\circ} \mathrm{C}$, the flow rate was $1.0 \mathrm{~mL} \mathrm{~min}^{-1}$, the injected volume was $20.0 \mu \mathrm{L}$ and the detection wavelength was set at $201 \mathrm{~nm}$. The developed and validated analytical method complies with the requirements established by the National Agency for Sanitary Vigilance in Brazil (ANVISA). ${ }^{24}$

Preparation of sample solutions, construction of linear analytical curves, determination of limits of detection (LOD) and quantification (LOQ)

The analytical solutions were prepared by dissolving $1.0 \mathrm{mg}$ of the samples plus $0.5 \mathrm{mg}$ of manool (MO), used as internal standard in $5 \mathrm{~mL}$ of acetonitrile, followed by filtration through a UNIFLO 25/0.2 PTFE (polytetrafluoroethylene) syringe filter (Whatman/ Schleicher \& Schuell, Maidstone, UK).
A dilution series of metabolites 1-6 (500.0, 400.0, 300.0, 200.0, 100.0, 80.0, 40.0, and $20.0 \mu \mathrm{g} \mathrm{mL} \mathrm{m}^{-1}$ ) with MO $\left(100.0 \mu \mathrm{g} \mathrm{mL}^{-1}\right)$ was prepared in acetonitrile. Then, $20.0 \mu \mathrm{L}$ aliquots of these solutions were injected into the HPLC equipment, in triplicate. Linear analytical curves were obtained by plotting the area ratio of the individual chromatographic standards to the internal standard MO. The correlation coefficient was calculated using Microsoft Excel $^{\circledR}$.

LOD and LOQ values were determined on the basis of the standard deviation of the response $(\sigma)$ and of the slope of the analytical curve $(\mathrm{S})$, using the expressions $\mathrm{LOD}=3.3 \sigma / \mathrm{S}$ and $\mathrm{LOQ}=10 \sigma / \mathrm{S}$.

\section{Selectivity}

The selectivity of the method was evaluated by comparing DAD (diode array detector) spectral data in the ascending, upper, and descending regions of the respective peaks of compounds 1-6 in the linear analytical curve and in the samples. ${ }^{9}$ All the spectra matched, confirming that no other metabolites co-eluted with the target compounds.

\section{Precision and accuracy}

The intra-day precision was evaluated by analyzing six samples, and the inter-day precision was assayed on two consecutive days. The concentrations and the relative standard deviations (RSD) of 1-6 were determined in their respective non-volatile ethyl acetate diterpenes soluble fractions, as follows: $\mathbf{1}, \mathbf{2}$ and $\mathbf{3}$ in NVCd; $\mathbf{1}$ in $\mathrm{NVCr}$; 4, 5 and 6 in NVCm. For the inter-day precision, the concentration values of compounds 1-6 were compared by applying the $t$-test $(p<0.05)$.

The accuracy of the method was determined by spiking known contents of compounds 1-6 into their respective oleoresins at low, medium and high levels (respectively, 25, 50 , and $100 \%$ of the target compounds content determined in the precision experiment), in triplicate. The spiked samples were analyzed and the recoveries were calculated by comparing the measured concentration to the spiked concentrations. ${ }^{25,26}$

\section{Robustness}

The robustness of the chromatographic method was assessed by following the experimental design proposed by Plackett-Burman for seven factors and eight experiments, as previously described. ${ }^{25,27}$ Briefly, the selected operational factors related to the chromatographic method were: mobile phase flow rate (Flow), column oven temperature (Temp.), percentage of organic solvent in the mobile phase (B, in $\%$ ), detection wavelength $(\lambda)$, and volume of injected samples (Inj.). Two dummy factors had to be included to 
reach a saturated design and variation levels for each of these operational factors (Table 1). Robustness values were expressed as RSD (\%) of the responses.

Table 1. Selected operational factors and variation levels related to the chromatographic method

\begin{tabular}{lcccc}
\hline Factor & Limit $( \pm)$ & Level $(-1)$ & Level $(+1)$ & Nominal \\
\hline Flow / $\left(\mathrm{mL} \mathrm{min}^{-1}\right)$ & 0.1 & 0.9 & 1.1 & 1.0 \\
Temp. $/{ }^{\circ} \mathrm{C}$ & 5 & 35.0 & 45.0 & 40.0 \\
$\mathrm{~B} / \%$ & 1 & 79.0 & 81.0 & 80.0 \\
$\lambda / \mathrm{nm}$ & 5 & 196 & 206 & 201 \\
Inj. / $\mu \mathrm{L}$ & 1 & 19.0 & 21.0 & 20.0 \\
\hline
\end{tabular}

Temp.: temperature; B: percentage of organic solvent in the mobile phase; $\lambda$ : detection wavelength; Inj.: volume of injected samples.

\section{Results and Discussion}

Compounds 1-6 (Figure 1) were isolated as main diterpenes from $\mathrm{OCd}, \mathrm{OCm}$ and $\mathrm{OCr}$, and their ${ }^{1} \mathrm{H}$ and ${ }^{13} \mathrm{C}$ NMR spectroscopic data are in agreement with those previously reported in the literature: (-)-polyalthic acid (1), ${ }^{28}$ (13E)-ent-labda-7,13-dien15-oic acid (2), ${ }^{29}$ ent-8(17)-labden-15,18-dioic acid (3), ${ }^{30}$ (-)-copalic acid (4), ${ }^{31}(-)-3 \beta$-acetoxycopalic acid $(\mathbf{5}),{ }^{32}$ (-)-3ß-hydroxycopalic acid (6). ${ }^{33}$ These compounds were evaluated by thin layer chromatography (TLC) using different solvent systems. According to this procedure and in addition to their spectroscopic data, the purities of these metabolites were estimated to be between $95-98 \%$.

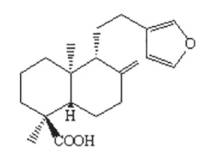

1

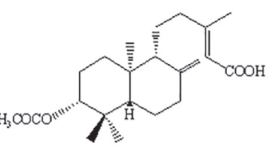

5
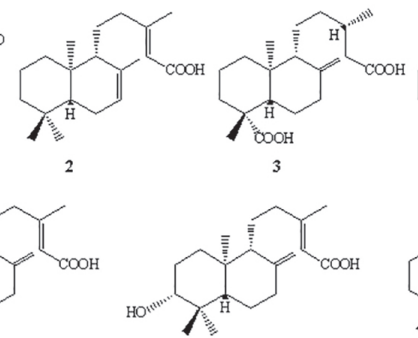
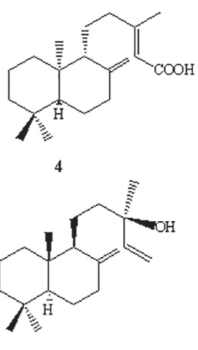

IS
Figure 1. Chemical structures of diterpenes isolated from $\mathrm{OCd}, \mathrm{OCm}$ and OCr and of the internal standard (IS) manool.

As previously reported, Copaifera oleoresins are sources of natural compounds with relevance for pharmaceutical and cosmetic applications. ${ }^{4,5,34}$ Despite their importance, many reports concerning the valuable properties of these balsams have not provided the proper botanical identification and/or a complete chemical characterization. As mentioned before, most of the previous reported studies had just carried out the chemical characterization of the volatile constituents, ${ }^{2,16-20}$ and have described the presence of more than 70 sesquiterpenes. ${ }^{2,9,10,35}$
Despite the importance of sesquiterpenes on the chemical and biological characterization of Copaifera oleoresins, ${ }^{1-3,17-19}$ recent reports have established that several non-volatile diterpenes, such as (-)-copalic acid, ent-kaurenoic acid and (-)-polyalthic acid, are also responsible for important biological properties displayed by these balsams. ${ }^{3,11,12,14,15,22}$ Even though and as stated before, there are only two analytical methods to characterize these diterpene acids in Copaifera oleoresins. ${ }^{9,21}$

Concerning to diterpenes, the literature ${ }^{11,17,36}$ reported that (-)-copalic acid (4) can be found in all oleoresins of Copaifera species and that it has been proposed as the chemical marker of the genus. However, our results are not in agreement with this report, since we have isolated only the (-)-copalic acid positional isomer (2) from $\mathrm{OCd}$, and a mixture of $\mathbf{2}+\mathbf{4}$ from OCr. It is important to observe that our research group has recently described the occurrence of $\mathbf{2}$ for the first time in Copaifera oleoresins, ${ }^{22}$ and due to the structural similarity between (-)-copalic (4) acid and its isomer (2), it is possible to suggest that the analytical methods previously reported are not able to distinguish between them. All these facts led to conclude that (-)-copalic acid should not be considered the only chemical marker of copaiba oleoresins.

Nowadays, the chemical fingerprints of herbs obtained by chromatographic methods have gained spotlight in the modern scientific literature, due to its importance as a powerful tool for authentication and application in the studies of natural products. ${ }^{37-39}$ Additionally, the World Health Organization (WHO) and several international regulatory agencies, such as the European Medicines Agency (EMEA), the State Food and Drug Administration of China (SFDA), and the Food and Drug Administration (FDA) of the United States of America have accepted the analysis of chemical markers for the evaluation of quality of medicinal plants, as well as their respective preparations..$^{37,38}$

Aiming to obtain a simple chemical profile focusing the diterpenes present in the oleoresins of C. duckei, C. reticulata and C. multijuga, it was firstly performed the hydrodistillation of $\mathrm{OCd}, \mathrm{OCr}$ and $\mathrm{OCm}$ using a Clevenger apparatus to separate the volatile and non-volatile fractions. It should be emphasized that the non-volatile diterpenes were recovered from the remaining water fraction after distillation by partition with ethyl acetate, in which all the components of the oleoresin are very soluble. This procedure was previously optimized by our research group, ${ }^{2,20}$ furnishing $72.6,7.2$ and $59.0 \%$ of the non-volatile fractions from C. duckei, C. multijuga and C. reticulata oleoresins, respectively. It is important to point out that this is a low cost, versatile, simple and rapid extraction 
procedure that can be easily employed in routine analysis of copaiba oleoresins and its products. ${ }^{2}$

The comparison among the obtained chromatograms for NVCd, NVCr, NVCm with those obtained for the authentic standards (compounds 1-6), allowed to observe that the non-volatile fractions of the three oleoresins display a simple chemical profile, as well as the presence of all isolated diterpenes in the phytochemical studies (Figure 2).

It can be depicted from Figure 2 that the developed analytical method allowed the identification of the main chromatographic peaks of each fraction, with exception of those at approximately 4.0 and $32.0 \mathrm{~min}$ in $\mathrm{NVCr}$ and

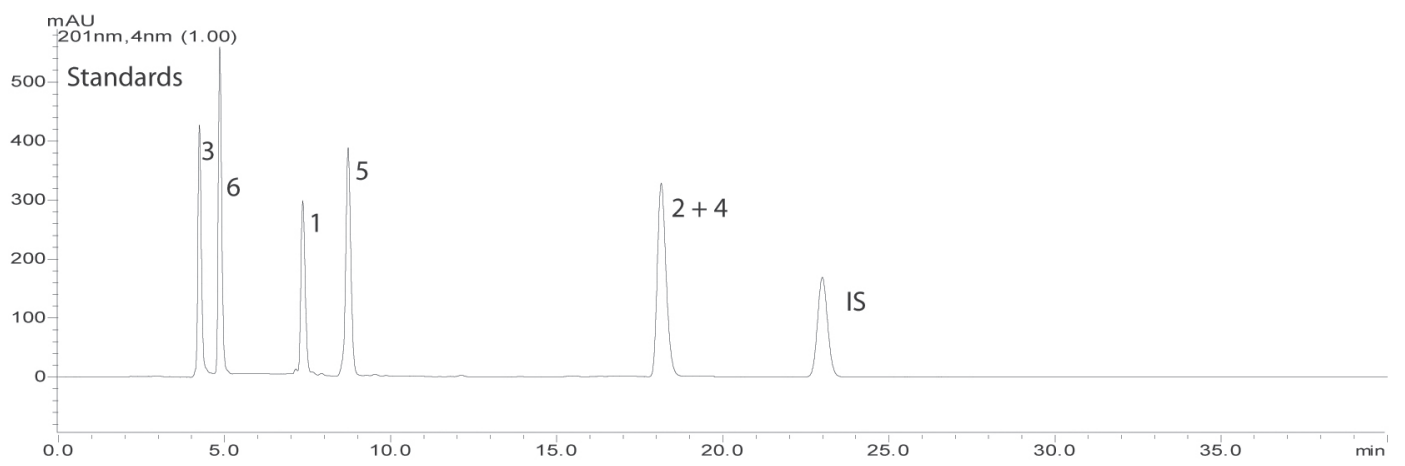

$500^{\mathrm{mAU}}{ }_{201 \mathrm{~nm}, 4 \mathrm{~nm}(1.00)}$
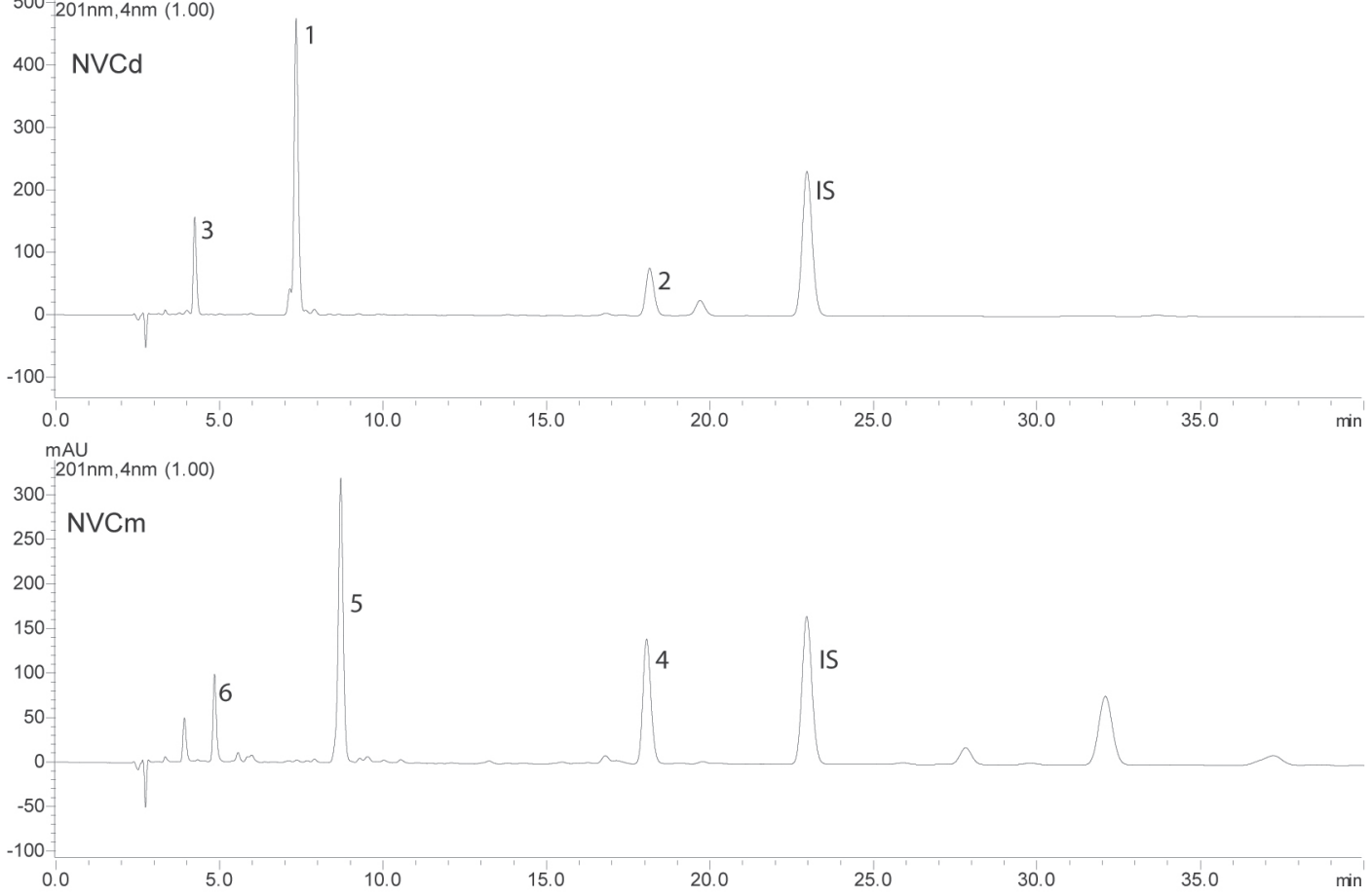

mAU

600-201nm, $4 \mathrm{~nm}(1.00)$

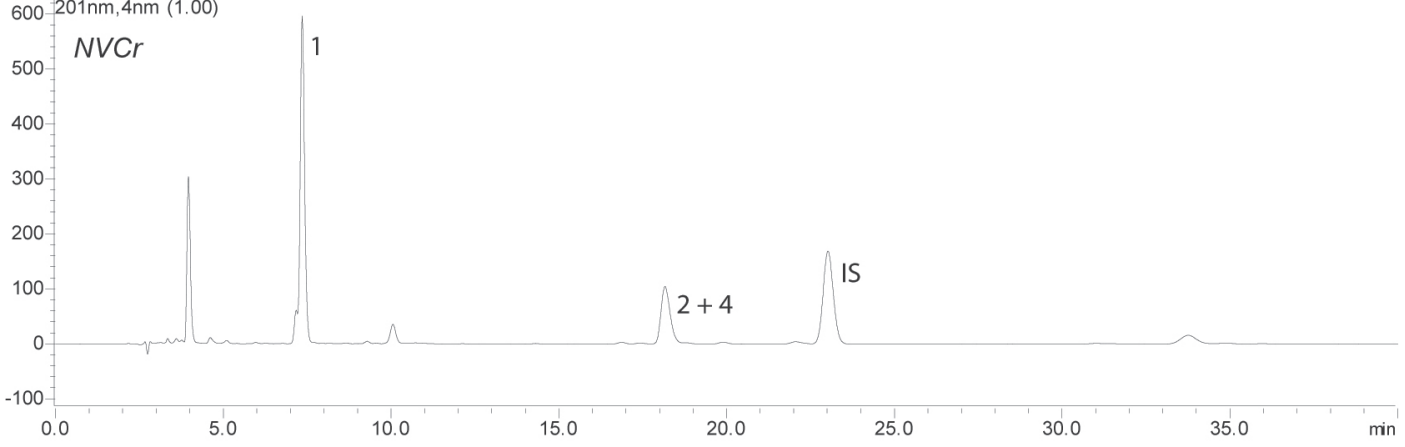

Figure 2. RP-HPLC chromatograms of NVCd, NVCr, NVCm and IS (manool) in comparison with the respective standards. (1) (-)-polyalthic acid; (2) (13E)-ent-labda-7,13-dien-15-oic acid; (3) ent-8(17)-labden-15,18-dioic acid; (4) (-)-copalic acid; (5) (-)-3ß-acetoxycopalic acid and (6) (-)-3ß-hydroxycopalic acid. 
NVCm, respectively. Also, the developed method does not allow to distinguish between (-)-copalic acid (4) and its positional isomer (2).

It is noteworthy that the oleoresins of $C$. duckei, C. reticulata and C. multijuga have a unique pattern based on their non-volatile diterpenes, and that the RP-HPLC fingerprints obtained for NVCd, $\mathrm{NVCr}$ and $\mathrm{NVCm}$ (Figure 2) allowed to clearly differentiate the three Brazilian species of Copaifera employed in this study. Moreover, the obtained results show that the chemical profiles of the non-volatile fractions from C. duckei, C. reticulata and C. multijuga are simple and much more specie-specific than their volatile compounds. Ideally, the selection of chemical markers of medicinal herbs should include bioactive metabolites. ${ }^{38}$ In this regard, the biological potential of the main diterpene constituents of these three oleoresins: (-)-polyalthic acid (1), (-)-copalic acid (4), (-)-3ß-acetoxycopalic acid (5) and (-)-3ß-hydroxycopalic acid have been very well established in the scientific literature. ${ }^{3,6,11,14,22}$

Therefore, non-volatile diterpenes are relevant and should be further employed in the authentication and quality control of Copaifera oleoresins and its preparations. However, more phytochemical and analytical studies focusing on the non-volatile compounds of other authentic species of Copaifera, as well as studies on seasonal and regional variations related to the Copaifera diterpenes should be stimulated. This would allow establishing the importance of this class of natural products for the quality control and authentication of these important oleoresins for pharmaceutical and cosmetic industries applications.

Finally, it is noteworthy that each oleoresin has a particular chemical profile (Figure 2), thus bringing new perspective for the RP-HPLC analyses of non-volatile compounds for authentication and quality control of Copaifera oleoresins and its preparations.

It is important to point out that the identified compounds comprise the majority of the non-volatile fractions of the oleoresins. The sum of the content of $\mathbf{1}, \mathbf{2}$, and $\mathbf{3}$ corresponds to approximately $80 \%$ of NVCd (Table 2). As for $\mathrm{NVCr}$, compound $\mathbf{1}$ is the major compound representing approximately $55 \%$ of the oleoresin mass. Finally, the total weight percent of $\mathbf{4}, \mathbf{5}$, and $\mathbf{6}$ corresponds to $78 \%$ of the NVCm mass. Considering the total mass of the oleoresins, the content of the identified diterpenes can be considered high for both OCd (58\%) and $\mathrm{OCr}(32 \%)$, and low for $\mathrm{OCm}(5 \%)$.

The analytical curves of standards 1-6 proved to be linear over the proposed range $\left(20.0-500.0 \mu \mathrm{g} \mathrm{mL}^{-1}\right)$, since they presented linear regression coefficients $\left(\mathrm{r}^{2}\right)$ ranging between 0.9903 and 0.9999 , thus denoting adequate data fits to their respective regression lines. The method also showed to be sensitive enough for all the target compounds, displaying LOD and LOQ values from 0.35 up to $3.09 \mu \mathrm{g} \mathrm{mL}^{-1}$ and from 1.05 to $9.36 \mu \mathrm{g} \mathrm{mL}^{-1}$, respectively (Table 3).

The developed method displayed good accuracy at low, medium and high levels for compounds 1-6, by displaying good recovery values (between 93.93 and $107.21 \%$ ), with RSD values lower than $2.0 \%$ (Table 4 ). ${ }^{40}$ It also showed RSD values lower than $2.0 \%$ in both the intra- and inter-day studies, thus denoting that the method is precise (Table 5).

As mentioned before, the robustness of the method was analyzed by examining the sources that are potentially subject to variations, when one or a set of responses inherent to the method are evaluated. Therefore, a number of factors into the validation procedure were selected and submitted to relatively mild variations. In general, these variations aimed to define the possibility of the given oscillation, when conducting the method on instruments of other brands or transferring it to another laboratory ${ }^{9,26}$ Responses "rrt" (relative retention time) and "concentration", as well as all other factors were considered to calculate the effects (Ex), which were then converted to RSD\% and analyzed (Table 6).

Table 2. Weight percent of identified compounds in their respective oleoresins and non-volatile fractions

\begin{tabular}{|c|c|c|c|c|c|c|}
\hline \multirow[t]{2}{*}{ Compound } & \multicolumn{2}{|c|}{ C. duckei / wt. $\%$} & \multicolumn{2}{|c|}{ C. multijuga / wt. $\%$} & \multicolumn{2}{|c|}{ C. reticulata / wt. $\%$} \\
\hline & $\mathrm{OCd}^{\mathrm{a}}$ & $\mathrm{NVCd}^{\mathrm{b}}$ & $\mathrm{OCm}^{\mathrm{a}}$ & $\mathrm{NVCm}^{\mathrm{b}}$ & $\mathrm{OCr}^{\mathrm{a}}$ & $\mathrm{NVCr}^{\mathrm{b}}$ \\
\hline 1 & 40.86 & 56.29 & & & 32.75 & 55.51 \\
\hline 2 & 9.35 & 12.88 & & & & \\
\hline 3 & 8.16 & 11.24 & & & & \\
\hline 4 & & & 1.89 & 26.28 & & \\
\hline 5 & & & 3.08 & 42.87 & & \\
\hline 6 & & & 0.69 & 9.62 & & \\
\hline
\end{tabular}

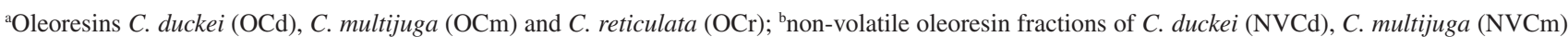
and C. reticulata (NVCr). 1: (-)-polyalthic acid; 2: (13E)-ent-labda-7,13-dien-15-oic acid; 3: ent-8(17)-labden-15,18-dioic acid; 4: (-)-copalic acid;

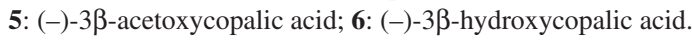


Table 3. Linear regression results, limits of detection (LOD) and quantification (LOQ)

\begin{tabular}{lcccc}
\hline Compound & Analytical equation & $\mathrm{r}^{2}$ & $\mathrm{LOD} /\left(\mu \mathrm{g} \mathrm{mL}{ }^{-1}\right)$ & $\mathrm{LOQ} /(\mu \mathrm{g} \mathrm{mL}-1)$ \\
\hline $\mathbf{1}$ & $\mathrm{y}=0.0067 \mathrm{x}-0.0038$ & 0.9995 & 3.09 & 9.36 \\
$\mathbf{2}$ & $\mathrm{y}=0.0108 \mathrm{x}-0.0198$ & 0.9999 & 0.41 & 1.25 \\
$\mathbf{3}$ & $\mathrm{y}=0.0069 \mathrm{x}+0.0587$ & 0.9936 & 0.93 & 5.84 \\
$\mathbf{4}$ & $\mathrm{y}=0.0049 \mathrm{x}-0.0194$ & 0.9993 & 0.49 & 1.05 \\
$\mathbf{5}$ & $\mathrm{y}=0.0122 \mathrm{x}-0.0631$ & 0.9997 & 0.70 & 1.49 \\
$\mathbf{6}$ & $\mathrm{y}=0.013 \mathrm{x}-0.191$ & 0.9903 & 2.13 \\
\hline
\end{tabular}

1: (-)-polyalthic acid; 2: (13E)-ent-labda-7,13-dien-15-oic acid; 3: ent-8(17)-labden-15,18-dioic acid; 4: (-)-copalic acid; 5: (-)-3ß-acetoxycopalic acid; 6: (-)-3ß-hydroxycopalic acid; $\mathrm{r}^{2}$ : coefficient of regression; LOD: limit of detection; LOQ: limit of quantification.

Table 4. Accuracy parameters expressed in percentage

\begin{tabular}{|c|c|c|c|c|c|c|c|c|c|c|}
\hline \multirow{2}{*}{ Compound } & \multirow{2}{*}{ Level } & \multicolumn{3}{|c|}{$\mathrm{NVCd}^{\mathrm{a}} / \%$} & \multicolumn{3}{|c|}{$\mathrm{NVCm}^{\mathrm{a}} / \%$} & \multicolumn{3}{|c|}{$\mathrm{NVCr}^{\mathrm{a}} / \%$} \\
\hline & & Recovery & Mean & RSD & Recovery & Mean & RSD & Recovery & Mean & RSD \\
\hline \multirow[t]{3}{*}{1} & 25 & 105.55 & 105.80 & 0.22 & & & & 96.52 & 95.26 & 1.36 \\
\hline & 50 & 105.84 & & & & & & 95.34 & & \\
\hline & 100 & 106.01 & & & & & & 93.93 & & \\
\hline \multirow[t]{3}{*}{2} & 25 & 101.41 & 101.48 & 0.55 & & & & & & \\
\hline & 50 & 102.07 & & & & & & & & \\
\hline & 100 & 100.96 & & & & & & & & \\
\hline \multirow[t]{3}{*}{3} & 25 & 100.38 & 99.17 & 1.17 & & & & & & \\
\hline & 50 & 98.06 & & & & & & & & \\
\hline & 100 & 99.35 & & & & & & & & \\
\hline \multirow[t]{3}{*}{4} & 25 & & & & 102.18 & 101.62 & 0.60 & & & \\
\hline & 50 & & & & 100.96 & & & & & \\
\hline & 100 & & & & 101.70 & & & & & \\
\hline \multirow[t]{3}{*}{5} & 25 & & & & 97.08 & 97.31 & 1.65 & & & \\
\hline & 50 & & & & 99.02 & & & & & \\
\hline & 100 & & & & 95.83 & & & & & \\
\hline \multirow[t]{3}{*}{6} & 25 & & & & 104.49 & 105.17 & 1.71 & & & \\
\hline & 50 & & & & 103.80 & & & & & \\
\hline & 100 & & & & 107.21 & & & & & \\
\hline
\end{tabular}

a Non-volatile oleoresin fractions of $C$. duckei (NVCd), C. multijuga (NVCm) and C. reticulata (NVCr). 1: (-)-polyalthic acid; 2: (13E)-ent-labda-7,13-dien-

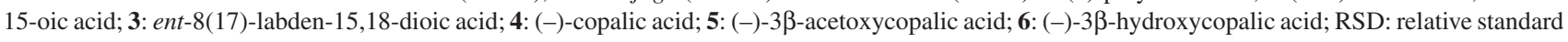
deviation.

Table 5. Results for intra- and inter-day precision (average of RSD\%)

\begin{tabular}{|c|c|c|c|c|c|c|}
\hline \multirow{3}{*}{ Compound } & \multicolumn{6}{|c|}{ Average of RSD / \% } \\
\hline & \multicolumn{2}{|c|}{$\mathrm{NVCd}^{\mathrm{a}}$} & \multicolumn{2}{|c|}{$\mathrm{NVCm}^{\mathrm{a}}$} & \multicolumn{2}{|c|}{$\mathrm{NVCr}^{\mathrm{a}}$} \\
\hline & Intra-day & Inter-day & Intra-day & Inter-day & Intra-day & Inter-day \\
\hline 1 & 1.37 & 1.60 & & & 1.40 & 1.18 \\
\hline 2 & 0.10 & 0.17 & & & & \\
\hline 3 & 0.19 & 0.15 & & & & \\
\hline 4 & & & 0.16 & 0.12 & & \\
\hline 5 & & & 0.12 & 0.11 & & \\
\hline 6 & & & 0.63 & 0.62 & & \\
\hline
\end{tabular}

${ }^{a}$ Non-volatile oleoresin fractions of $C$. duckei (NVCd), C. multijuga (NVCm) and C. reticulata (NVCr). 1: (-)-polyalthic acid; 2: (13E)-ent-labda-7,13-dien-

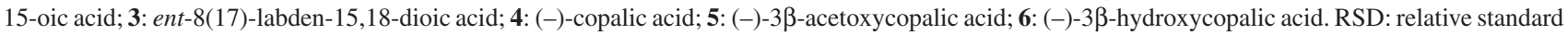
deviation. 
Table 6. RSD values obtained for the robustness studies

\begin{tabular}{|c|c|c|c|c|c|c|c|c|c|c|c|c|c|c|}
\hline \multirow{4}{*}{ Parameter } & \multicolumn{14}{|c|}{$\mathrm{RSD} / \%$} \\
\hline & \multicolumn{6}{|c|}{$\mathrm{NVCd}^{\mathrm{a}}$ compound } & \multicolumn{6}{|c|}{$\mathrm{NVCm}^{\mathrm{a}}$ compound } & \multirow{2}{*}{\multicolumn{2}{|c|}{$\frac{\mathrm{NVCr}^{\mathrm{a}} \text { compound }}{\mathbf{1}}$}} \\
\hline & \multicolumn{2}{|c|}{1} & \multicolumn{2}{|c|}{2} & \multicolumn{2}{|c|}{3} & \multicolumn{2}{|r|}{4} & \multicolumn{2}{|c|}{5} & \multicolumn{2}{|r|}{6} & & \\
\hline & rrt & $\begin{array}{c}\text { Concen- } \\
\text { tration }\end{array}$ & rrt & $\begin{array}{l}\text { Concen- } \\
\text { tration }\end{array}$ & rrt & $\begin{array}{c}\text { Concen- } \\
\text { tration }\end{array}$ & rrt & $\begin{array}{c}\text { Concen- } \\
\text { tration }\end{array}$ & rrt & $\begin{array}{c}\text { Concen- } \\
\text { tration }\end{array}$ & $\mathrm{rrt}$ & $\begin{array}{l}\text { Concen- } \\
\text { tration }\end{array}$ & $\mathrm{rrt}$ & $\begin{array}{c}\text { Concen- } \\
\text { tration }\end{array}$ \\
\hline Flow & 0.00 & -0.95 & 0.07 & -0.78 & 0.07 & -0.10 & 0.08 & -0.03 & 0.09 & -0.74 & -0.08 & -0.09 & 0.05 & 0.17 \\
\hline Temp. & -0.99 & 1.63 & -0.95 & 5.29 & -0.91 & 0.38 & -0.95 & 0.66 & 0.83 & 0.58 & -1.07 & -0.51 & -0.97 & 0.19 \\
\hline B & 0.06 & -0.53 & 0.05 & -2.51 & 0.14 & 0.18 & 0.07 & 0.06 & 1.16 & 1.16 & -0.41 & 0.90 & 0.12 & -0.69 \\
\hline$\lambda$ & -0.20 & 51.15 & -0.19 & 46.02 & -0.12 & 29.51 & -0.18 & 48.84 & -0.64 & 36.87 & -0.84 & 39.17 & -0.18 & 51.11 \\
\hline Inj. & -0.08 & 0.11 & -0.01 & 1.84 & -0.03 & -0.32 & 0.00 & -0.58 & -0.10 & -1.26 & -0.15 & -0.23 & 0.01 & -0.11 \\
\hline
\end{tabular}

aNon-volatile oleoresin fractions of C. duckei (NVCd), C. multijuga $(\mathrm{NVCm})$ and C. reticulata $(\mathrm{NVCr}) ; 1$ : (-)-polyalthic acid; 2: (13E)-ent-labda-7,13-dien-15-oic

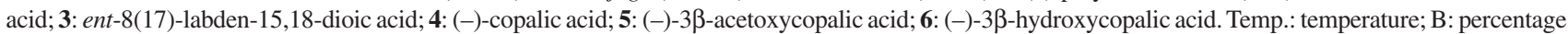
of organic solvent in the mobile phase; $\lambda$ : detection wavelength; Inj.: volume of injected samples; RSD: relative standard deviation; rrt: relative retention time.

When considering these effects, the most prominent RSD values were related to the influence of the wavelength detection $(\lambda)$ in the "concentration" response. In this regard, small variations in the wavelength elicit pronounced changes in the absorbance, ranging between 29.51 and $51.15 \%$. However, the overall assessment of the obtained results allowed to consider the developed method suitable to be used in different laboratories..$^{9,26}$

\section{Conclusions}

The reported RP-HPLC-PDA analytical method allowed the identification and quantification of six diterpenes present in three non-volatile oleoresin fractions derived from Copaifera oleoresins. Also, the chemical profiles of these diterpenes in C. duckei, C. reticulata and C. multijuga were simpler and much more species-specific than their volatile compounds. All these facts led us to conclude that the developed analytical method is simple, reliable and should be considered to be used routinely in the analyses of Copaifera oleoresins and its products, as well as in other studies, since it met the requirements of the Brazilian Regulatory Agency.

\section{Supplementary Information}

Supplementary data (NMR and UV spectra) are available free of charge at http://jbcs.sbq.org.br as PDF file.

\section{Acknowledgments}

The authors are grateful to the State of São Paulo Research Foundation (FAPESP) for financial support, grants number 2011/13630-7, 2014/03092-6 and 2016/01232-0, as well as to CAPES and CNPq for fellowships.

\section{References}

1. Borges, V. R. A.; Ribeiro, A. F.; Anselmo, C. S.; Cabral, L. M.; de Sousa, V. P.; J. Chromatogr. B 2013, 940, 35.

2. Sousa, J. P. B.; Brancalion, A. P. S.; Souza, A. B.; Turatti, I. C. C.; Ambrósio, S. R.; Furtado, N. A. J. C.; Lopes, N. P.; Bastos, J. K.; J. Pharm. Biomed. Anal. 2011, 54, 653.

3. Leandro, L. M.; Vargas, F. D.; Barbosa, P. C. S.; Neves, J. K. O.; da Silva, J. A.; da Veiga, V. F.; Molecules 2012, 17, 3866.

4. Vargas, F. S.; de Almeida, P. D. O.; Aranha, E. S. P.; Boleti, A. P. A.; Newton, P.; de Vasconcellos, M. C.; Veiga Junior, V. F.; Lima, E. S.; Molecules 2015, 20, 6194.

5. Izumi, E.; Ueda-Nakamura, T.; Veiga Jr., V. F.; Pinto, A. C.; Nakamura, C. V.; J. Med. Chem. 2012, 55, 2994.

6. Souza, A. B.; de Souza, M. G. M.; Moreira, M. A.; Moreira, M. R.; Furtado, N. A. J. C.; Martins, C. H. G.; Bastos, J. K.; Santos, R. A.; Heleno, V. C. G.; Ambrosio, S. R.; Veneziani, R. C. S.; Molecules 2011, 16, 9611.

7. Tappin, M. R. R.; Pereira, J. F. G.; Lima, L. A.; Siani, A. C.; Mazzei, J. L.; Ramos, M. F. S.; Quim. Nova 2004, 27, 236.

8. Lima, S. R. M.; Veiga Jr., V. F.; Christo, H. B.; Pinto, A. C.; Fernandes, P. D.; Phytother. Res. 2003, 17, 1048.

9. Souza, A. B.; Moreira, M. R.; Borges, C. H. G.; Simão, M. R.; Bastos, J. K.; de Sousa, J. P. B.; Ambrosio, S. R.; Veneziani, R. C. S.; Biomed. Chromatogr. 2013, 27, 280.

10. Barbosa, P. C. S.; Medeiros, R. S.; Sampaio, P. T. B.; Vieira, G.; Wiedemann, L. S. M.; Veiga-Junior, V. F.; J. Braz. Chem. Soc. 2012, 23, 1823.

11. Souza, A. B.; Martins, C. H. G.; Souza, M. G. M.; Furtado, N. A. J. C.; Heleno, V. C. G.; Sousa, J. P. B.; Rocha, E. M. P.; Bastos, J. K.; Cunha, W. R.; Veneziani, R. C. S.; Ambrósio, S. R.; Phytother. Res. 2011, 25, 215.

12. Mizokami, S. S.; Arakawa, N. S.; Ambrosio, S. R.; Zarpelon, A. C.; Casagrande, R.; Cunha, T. M.; Ferreira, S. H.; Cunha, F. Q.; Verri, W. A.; J. Nat. Prod. 2012, 75, 896. 
13. Lizarte Neto, F. S.; Tirapelli, D. P. C.; Ambrosio, S. R.; Tirapelli, C. R.; Oliveira, F. M.; Novais, P. C.; Peria, F. M.; Oliveira, H. F.; Carlotti Junior, C. G.; Tirapelli, L. F.; Braz. J. Med. Biol. Res. 2013, 46, 71.

14. dos Santos, A. O.; Izumi, E.; Ueda-Nakamura, T.; Dias, B. P.; da Veiga, V. F.; Nakamura, C. V.; Mem. Inst. Oswaldo Cruz 2013, 108, 59 .

15. Simão, M. R.; Carneiro, L. J.; Santos, R. A.; Bastos, J. K.; Veneziani, R. C. S.; Ambrósio, S. R.; Mizuno, C. S.; Med. Chem. Res. 2015, 25, 303.

16. Veiga, V. F.; Zunino, L.; Calixto, J. B.; Patitucci, M. L.; Pinto, A. C.; Phytother. Res. 2001, 15, 476.

17. Veiga, V. F.; Rosas, E. C.; Carvalho, M. V.; Henriques, M. G. M. O.; Pinto, A. C.; J. Ethnopharmacol. 2007, 112, 248.

18. Lima, C. S.; de Medeiros, B. J. L.; Favacho, H. A. S.; dos Santos, K. C.; de Oliveira, B. R.; Taglialegna, J. C.; da Costa, E. V. M.; de Campos, K. J.; Carvalho, J. C. T.; Phytomedicine 2011, 18, 1013.

19. Lucca, L. G.; de Matos, S. P.; Borille, B. T.; Dias, D. O.; Teixeira, H. F.; Veiga Jr., V. F.; Limberger, R. P.; Koester, L. S.; J. Pharm. Biomed. Anal. 2015, 104, 144.

20. Bardají, D. K. R.; da Silva, J. J. M.; Bianchi, T. C.; Eugênio, D. S.; de Oliveira, P. F.; Leandro, L. F.; Rogez, H. L. G.; Venezianni, R. C. S.; Ambrosio, S. R.; Tavares, D. C.; Bastos, J. K.; Martins, C. H. G.; Anaerobe 2016, 40, 18.

21. da Silva, J. J. M.; Crevelin, E. J.; Carneiro, L. J.; Rogez, H.; Veneziani, R. C. S.; Ambrósio, S. R.; Beraldo Moraes, L. A.; Bastos, J. K.; J. Chromatogr. A 2017, 1515, 81.

22. Borges, C. H. G.; Cruz, M. G.; Carneiro, L. J.; da Silva, J. J. M.; Bastos, J. K.; Tavares, D. C.; de Oliveira, P. F.; Rodrigues, V.; Veneziani, R. C. S.; Parreira, R. L. T.; Caramori, G. F.; Nagurniak, G. R.; Magalhães, L. G.; Ambrósio, S. R.; Chem. Biodiversity 2016, 13, 1348.

23. Snyder, L. R.; Dolan, J. W.; J. Chromatogr. A 1996, 721, 3.

24. ANVISA, RE899; Guia para Validação de Métodos Analíticos e Bioanalíticos; ANVISA, Diário Oficial da União, Brasília, 2003.
25. Moreira, M. R.; Souza, A. B.; Soares, S.; Bianchi, T. C.; Eugenio, D. S.; Lemes, D. C.; Martins, C. H.; Moraes, T. S.; Tavares, D. C.; Ferreira, N. H.; Ambrosio, S. R.; Veneziani, R. C.; Fitoterapia 2016, 112, 211.

26. Moreira, M. R.; Souza, A. B.; Moreira, M. A.; Bianchi, T. C.; Carneiro, L. J.; Estrela, F. T.; Santos, R. A.; Januário, A. H.; Martins, C. H. G.; Ambrosio, S. R.; Veneziani, R. C. S.; Braz. J. Pharmacog. 2013, 23, 870.

27. Vander Heyden, Y.; Nijhuis, A.; Smeyers-Verbeke, J.; Vandeginste, B. G. M.; Massart, D. L.; J. Pharm. Biomed. Anal. 2001, 24, 723.

28. Carreras, C. R.; Rossomando, P. C.; Giordano, O. S.; Phytochemistry 1998, 48, 1031.

29. Xiang, W.; Li, R. T.; Song, Q. S.; Na, Z.; Sun, H. D.; Helv. Chim. Acta 2004, 87, 2860.

30. Henrick, C. A.; Jefferie, P.; Tetrahedron 1965, 21, 3219.

31. Ohsaki, A.; Yan, L. T.; Ito, S.; Edatsugi, H.; Iwata, D.; Komoda, Y.; Bioorg. Med. Chem. 1994, 4, 2889.

32. Braun, S.; Breitenbach, H.; Tetrahedron 1977, 33, 145.

33. Romero, A. L.; Baptistella, L. H. B.; Imamura, P. M.; J. Braz. Chem. Soc. 2009, 20, 1036.

34. Gelmini, F.; Beretta, G.; Anselmi, C.; Centini, M.; Magni, P.; Ruscica, M.; Cavalchini, A.; Facino, R. M.; Int. J. Pharm. 2013, 440, 170.

35. Herrero-Jauregui, C.; Casado, M. A.; Zoghbi, M. D. B.; Martinsda-Silva, R. C.; Chem. Biodiversity 2011, 8, 674.

36. Veiga, V. F.; Pinto, A. C.; Quim. Nova 2002, 25, 273.

37. Goodarzi, M.; Russell, P. J.; Vander Heyden, Y.; Anal. Chim. Acta 2013, 804, 16.

38. Masullo, M.; Montoro, P.; Mari, A.; Pizza, C.; Piacente, S.; J. Pharm. Biomed. Anal. 2015, 113, 189.

39. Aliakbarzadeh, G.; Sereshti, H.; Parastar, H.; Anal. Bioanal. Chem. 2016, 408, 3295.

40. Baccarin, T.; Muceneeki, R. S.; Bresolin, T. M.; Yunes, R. A.; Malheiros, A.; Lucinda-Silva, R. M.; Talanta 2011, 85, 1221.

Submitted: September 12, 2017

Published online: October 27, 2017 